\title{
Delayed Positivization of Cerebral Angiography in Reversible Cerebral Vasoconstriction Syndrome (RCVS) Presenting with Recurrent Subara- chnoid Haemorrhage
} \author{
Salvatore De Vita ${ }^{1}$ \\ ${ }^{I}$ Clinic of Rheumatology, Azienda Ospedaliero-Universitaria di Udine, DSMB, Italy \\ ${ }^{2}$ Department of Neurosurgery, Azienda Ospedaliero-Universitaria di Udine, Italy \\ ${ }^{3}$ Department of Neuroradiology, Azienda Ospedaliero-Universitaria di Udine, Italy
}

Luca Quartuccio $^{*}, 1, \S$, Francesco Tuniz ${ }^{2, \S}$, Benedetto Petralia ${ }^{3}$, Bruno Zanotti ${ }^{2}$, Miran Skrap ${ }^{2}$ and

\begin{abstract}
Benign angiopathy of the central nervous system is a clinical syndrome with evidence of reversible cerebral vasoconstriction (RCVS). Haemorrhagic stroke, either subarachnoid or intracerebral is an unusual presentation of RCVS. We describe a case of RCVS presenting with a subarachnoid haemorrhage (SAH), with rebleeding and onset of hydrocephalus during the first week, and, notably, delayed evidence of typical angiographic features after two negative prior exams. Normalization of the angiographic vasculitic-like lesions was documented at month +6 . Repeated cerebral angiograms are mandatory to exclude this kind of disease, and the uncommon presentation of this case reinforces this concept.
\end{abstract}

Keywords: Reversible cerebral vasoconstriction syndrome, angiography, hydrocephalus, subarachnoid haemorrhage, vasculitis.

\section{INTRODUCTION}

Vasculitis of the central nervous system (CNS) is classified as primary angiitis or as secondary vasculitis related to a variety of diseases [1]. Reversible cerebral vasoconstriction syndromes (RCVS) represent a critical differential diagnosis of CNS vasculitis and they comprise a group of disorders characterized by prolonged but reversible vasoconstriction of the cerebral arteries, usually associated with acute-onset, severe, recurrent headaches, with or without additional neurologic signs and symptoms [2]. We report a case of a female patient with initial suspicion of primary angiitis of the cerebral nervous system (PACNS) presenting with a subarachnoid haemorrhage (SAH). Delayed evidence of typical angiographic features after negative prior angiograms, and the disappearance of the abnormalities six month later led to revise the initial diagnosis of PACNS towards the diagnosis of RCVS. The occurrence of delayed positivization of the cerebral angiograms must be considered for the diagnosis of this kind of the disease and it may differentiate RCVS from PACNS.

\section{CASE REPORT}

A 47-year-old woman was admitted to the Emergency Department of Udine Hospital on June 2006 for a sudden onset of thunder-clap headache following by loss of

*Address correspondence to this author at the Clinic of Rheumatology, DSMB, University of Udine, Univerisyt Hospital "Santa Maria della Misericordia", Udine, Piazzale Santa Maria della Misericordia, 15, Italy; Tel: 00390432 559352; Fax: 00390432 559472;

E-mail: quartuccio.luca@aoud.sanita.fvg.it

${ }^{\S}$ L. Quartuccio and F. Tuniz equally collaborated to this work. consciousness. Cerebral computed tomography scan (CT) revealed a subarachnoid haemorrhage, thus the patient was soon transferred to the Neurosurgery Unit. At admission in neurosurgical ward the patient was awake, alert, apyretic and with mild arterial hypertension $(150 / 90 \mathrm{mmHg})$. No intracranial aneurysms or arterio-venous malformations $(\mathrm{AVM})$ were found on cerebral four vessels angiography performed the first day after admission (Fig. 1). The patient was treated with nifedipine, obtaining normalization of blood pressure values. At the entrance inflammatory markers, such as erythrocyte sedimentation rate (ESR), C-reactive protein (CRP), serum ferritin and fibrinogen were normal. Hemoglobin level, leukocyte and platelet count, hepatic and renal function tests, protein electrophoresis, $\mathrm{C} 3$ and $\mathrm{C} 4$ levels were normal.

Seven days later the patient experienced a worsening of her neurological status, she complained of a crushing headache and nausea, and she vomited several times. A CT scan was performed and showed a new perimesenchepalic bleeding and acute hydrocephalus. An external ventricular drainage was performed. After this surgical procedure the neurological status temporally improved.

The second cerebral four vessels angiography performed fourteen days after the first one as a control after the second cerebral haemorrhage, did not show any arterial abnormalities. In particular, no aneurysms or AVM were documented. In the following days the patient referred mild headaches, myalgias and even muscle weakness, and developed a slight right hemiparesis. Serial CT scans documented the progressive reabsorption of the subarachnoid haemorrhage. 


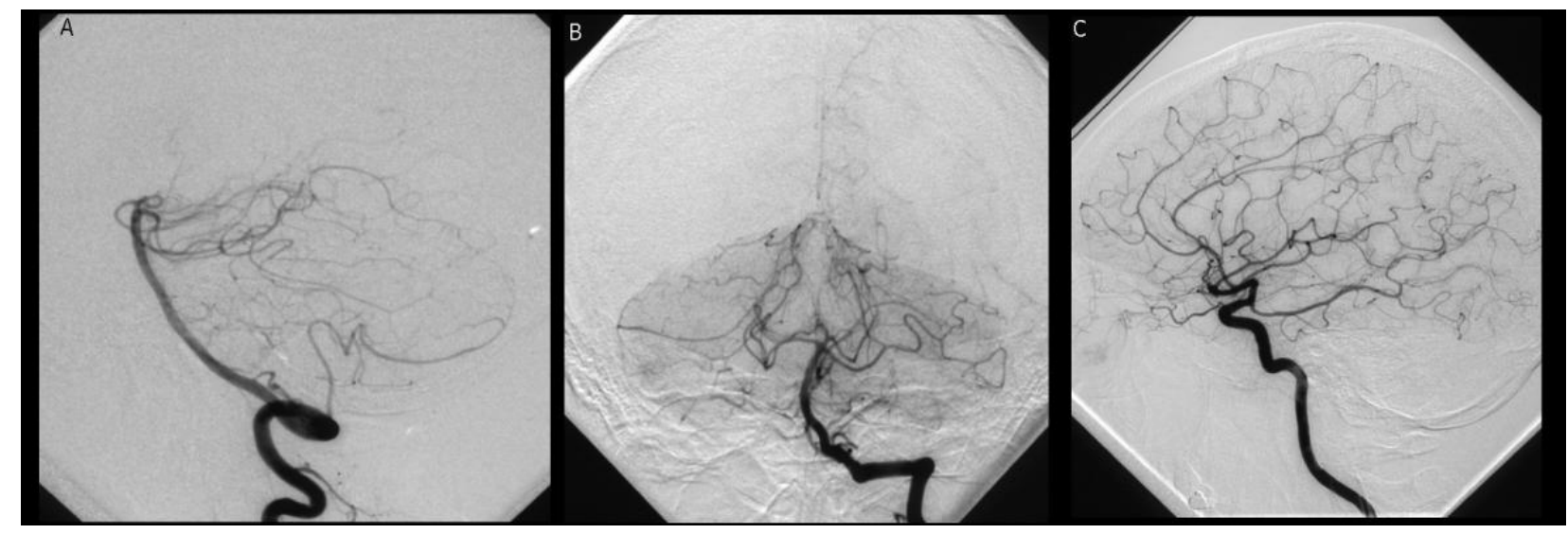

Fig. (1). Conventional angiography, first one, of the vertebrobasilar system (A, B) and the left carotid system (C): no arterial abnormalities, in particular no aneurysms or A-V malformation.

The third cerebral four vessels control angiography performed one month after the first one, (Fig. 2) showed marked focal narrowing and stenosis of both carotid siphons (supraclinoid tract), of the terminal tract of the basilar artery, of both P1-P2 tracts, and A1 right tract. No venous or capillar abnormalities were noticed. These findings, very delayed after the onset, excluded a vasospastic complication of SAH [3]. A ventriculo-peritoneal shunt was performed.

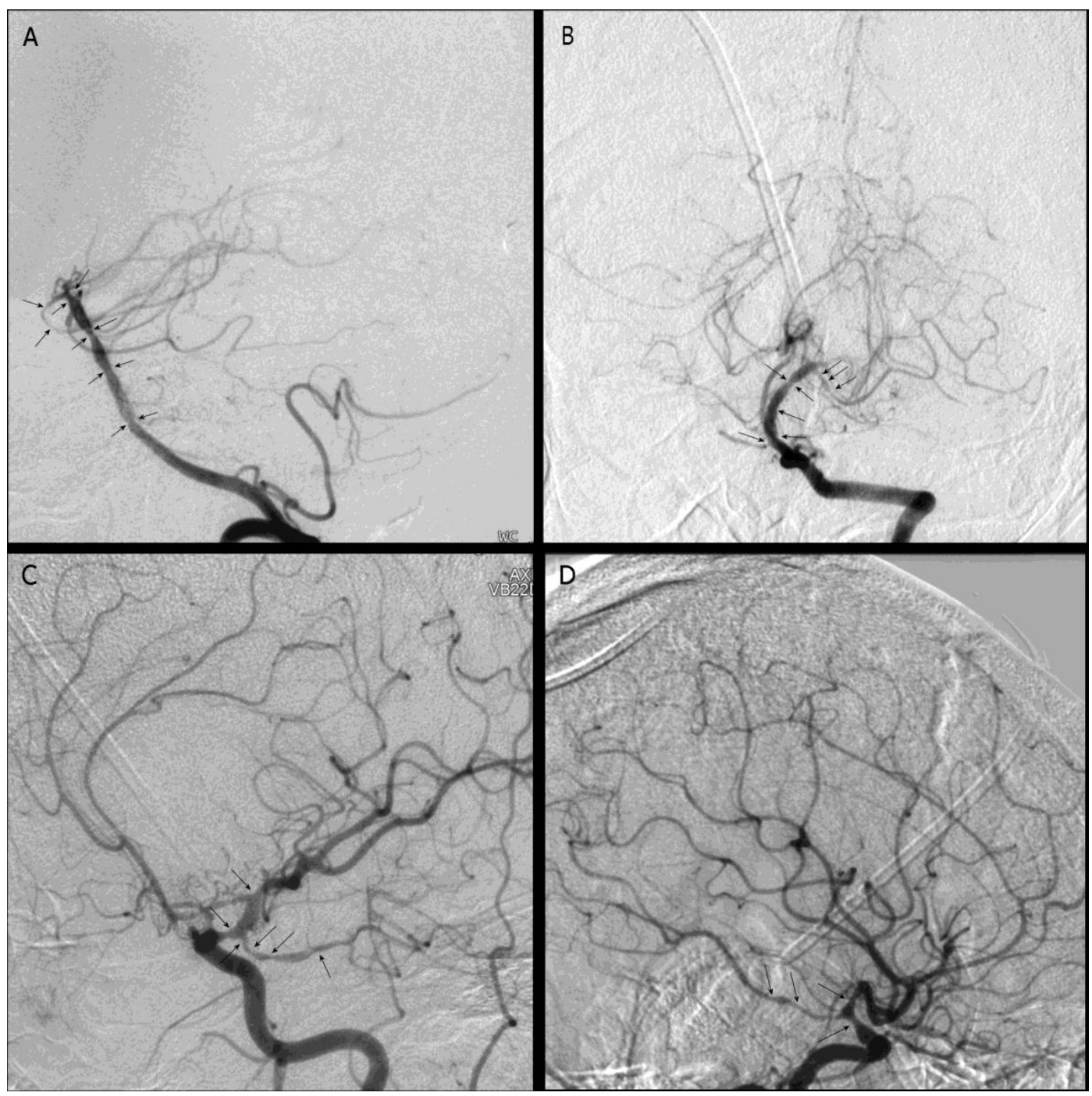

Fig. (2). Conventional angiography, one month after bleeding: marked focal narrowing and stenosis of both carotid siphons (arrows), terminal tract of the basilar artery, both P1-P2 tracts, and A1 right tract. 
Then, the patient was evaluated by the Rheumatologist consultant, who planned an intense workup to clarify the diagnosis of cerebral vasculitis. Cerebral vasculitis due to a systemic disease was excluded based on i) the patient's history and physical examination, not indicating an underlying systemic disease, such as systemic vasculitis, or connective tissue diseases; ii) the absence of serum antinuclear antibodies, anticytoplasmic neutrphil antibodies, rheumatoid factor, antiphospholipid antibodies and cryoglobulins. Inflammatory markers, such as ESR, CRP, serum ferritin and fibrinogen were normal. Cerebrospinal fluid (CSF) analysis resulted abnormal, showing lymphocytic pleocytosis and evidence of haemorrhage. Leptomeningeal biopsy was not performed due to the critical condition of the patient at the time of her admission in the Neurosurgery Unit. Based on these findings the diagnosis of PACNS was made and the patient started high-dose corticosteroids (dexamethasone $8 \mathrm{mg} \mathrm{x} \mathrm{3/day,} \mathrm{for} \mathrm{three}$ weeks, then $8 \mathrm{mg} \times 2 /$ day for one week, then $8 \mathrm{mg} /$ day for one week, then methylprednisolone $32 \mathrm{mg} /$ day, tapered to 6 $\mathrm{mg} /$ day in two weeks) and pulse intravenous cyclophosphamide ( $750 \mathrm{mg}$ weekly for 3 weeks, followed by $500 \mathrm{mg}$ weekly for 2 weeks). The treatment was well tolerated and no other cerebral haemorrhage episodes were observed. No maintenance immunosuppressive treatment was then employed. Subsequently, the patient completed her rehabilitation program. Steroids were tapered to $5 \mathrm{mg}$ /day of prednisone at month +6 and then maintained at this dose due to secondary hypopituitarism. A repeated cerebral angiography at month +6 did not reveal any abnormality with a complete vessels size recovery (Fig. 3). At the last follow-up (month +61 ) no relapse occurred, and the patient was able to normally make her daily practice activities. The cerebral MRI showed ischemic lesions in left thalamus, left pons and mesencephalon at month +6 (Fig. 4) and at month +12 and then remained unchanged in the subsequent annually repeated MRI.

\section{DISCUSSION}

The first step to diagnose CNS vasculitis is early suspicion, but all the non-vasculitic processes that might be involved should be excluded $[1,2]$. In our case, the patient's history, the physical exam, the laboratory findings and the instrumental tests excluded other non-vasculitic causes of
CNS haemorrhage, i.e. infectious diseases, uncontrolled arterial hypertension, malignancy, aneurism rupture, metabolic disorders, or drug abuse. Furthermore, a CNS involvement either in the context of a systemic vasculitis, such as Wegener's granulomatosis, giant cell arteritis, polyarteritis nodosa or cryoglobulinemia, or in the course of other rheumatic diseases, such as systemic lupus erythematosus, Sjögren's syndrome, rheumatoid arthritis, Behçet syndrome, sarcoidosis, was considered and excluded through a careful workup. The initial diagnosis of isolated CNS vasculitis was suggested only by the angiographic study, since the diagnostic brain biopsy and a magnetic resonance imaging study were not performed due to the critical condition of the patient and the CSF was not informative. Thus, the acute onset and the rapid recurrence of cerebral haemorrhage justified an initial aggressive immunosuppressive approach. However, the favourable outcome characterized by a rapid and sustained response after a short course of immunosuppression, CSF slightly abnormal, angiographic changes compatible with vasculitis suggested a benign angiopathy of the CNS, possibly with a vasospasm-induced angiopathy rather than a vasculitis $[2,4$, 5]. The disappearance of the abnormal findings in the repeated angiography at month +6 confirmed the benign nature of the disease. Based on a very favourable clinical response, the cyclophosphamide was stopped and steroids were minimized at the lowest dose, as soon as possible.

RCVS can be confused with cerebral vasculitis, and in unusual and acute presentation $[2,4,5]$, mainly when intracranial haemorrhage occurred [6]. This disorder is characterized by reversible multifocal narrowing of the cerebral arteries heralded by sudden, severe headaches with or without neurologic deficits, and it seems to well respond to a short course of glucocorticoids and calcium-channel blockers [2]. Typically, angiographic abnormalities resolved within 12 weeks [2]. In our case, the angiographic vasculiticlike findings developed later, when haemorrhage had already occurred, and were absent in the first two examinations. The delayed onset of angiographic vessel abnormalities excluded a vasospastic complication of SAH [3]. However, this delayed appearance of typical vessel abnormalities was quite unusual in RCVS [7], but angiogram-negative SAH is a well recognized and open issue, and it accounts for about $15 \%$ of nontraumatic SAH [8]. Also, postpartum cerebral angiopathy

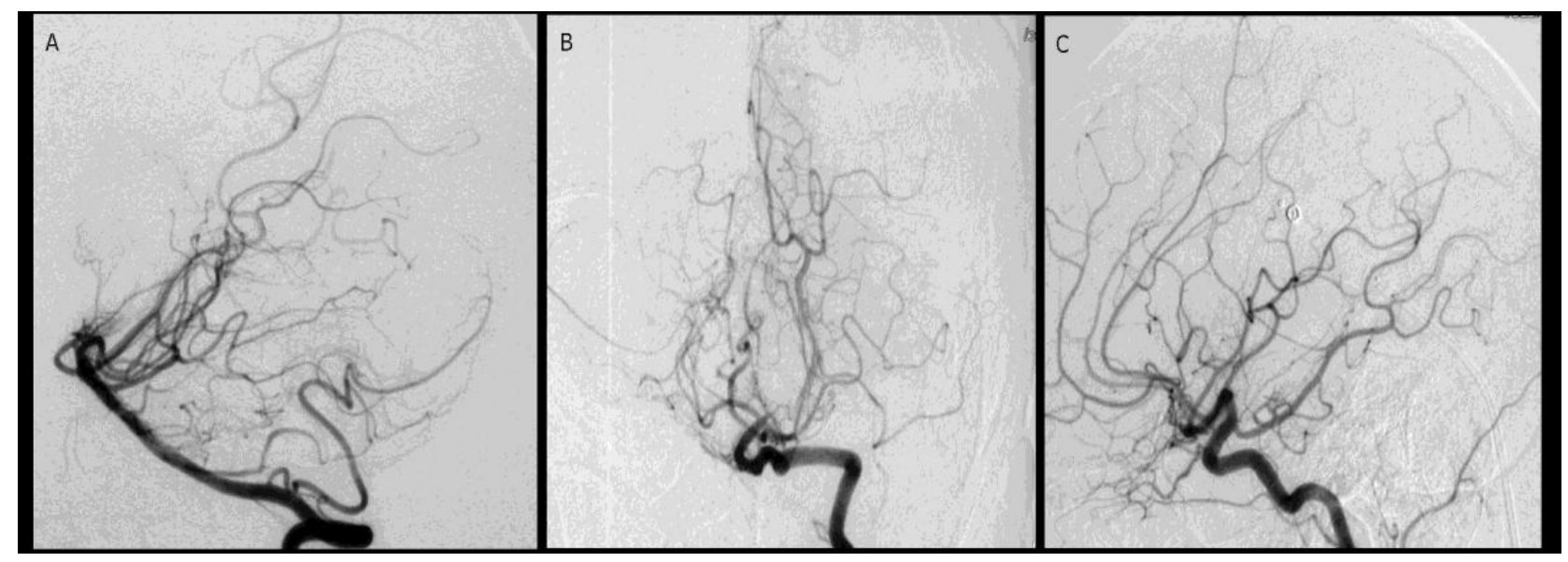

Fig. (3). Conventional angiography, six months after bleeding and after immunosuppressive treatment. 


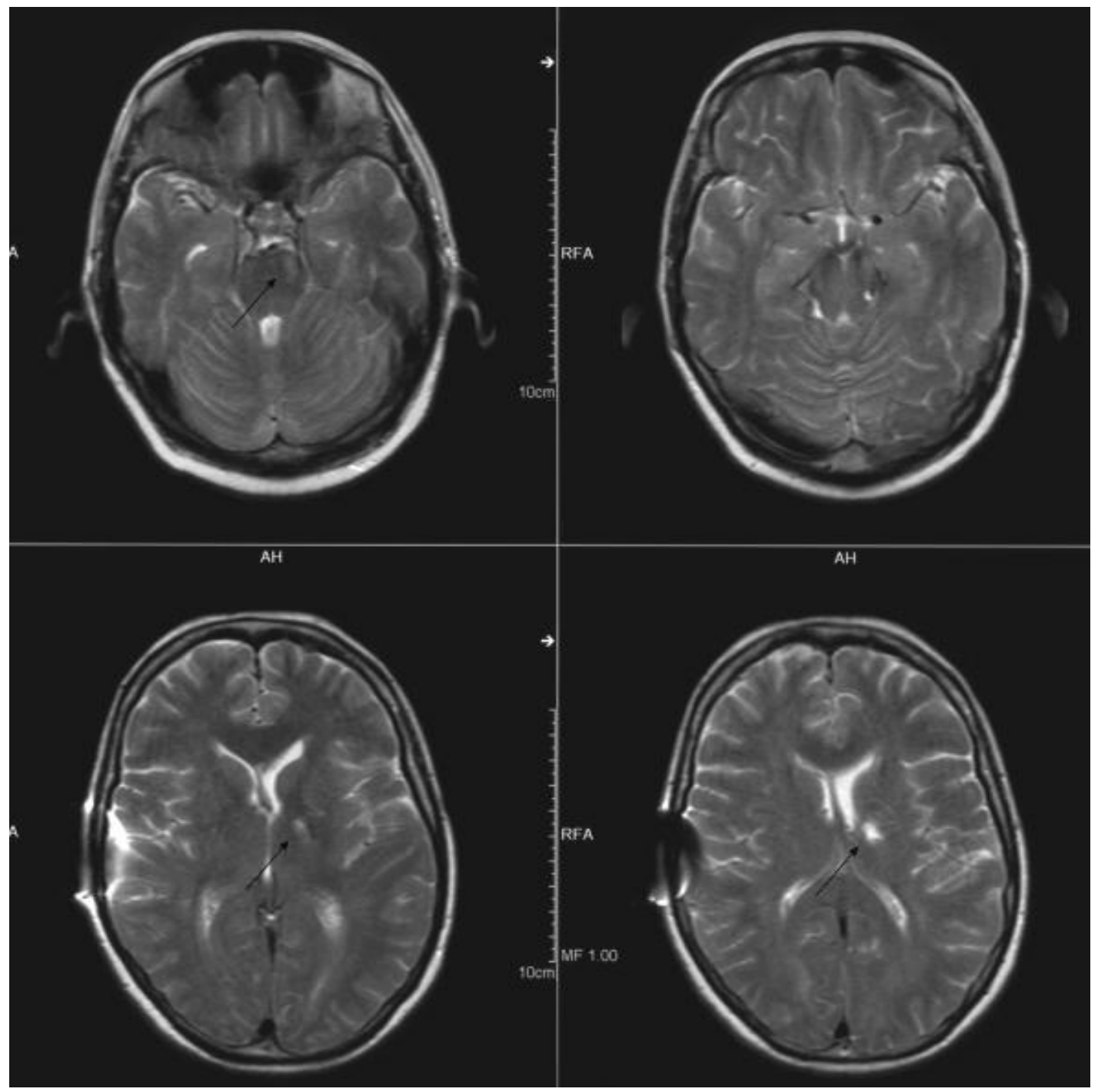

Fig. (4). Cerebral MRI six months after bleeding: ischemic lesions in left thalamus, left pons and mesencephalon (arrows).

represents a well known subgroup of the $\operatorname{RCVS}[9,10]$, where there is an increasingly description of cases with a delay between clinical onset and angiographic changes [11]. It has been suggested a sequential involvement of arterial beds, initial involvement of small distal arteries (not visible on angiography) progressing over days or weeks to involve medium and large sized arteries (visible by angiography) [12].

Given the small size of the reported group and the absence of a prospective randomized controlled therapeutic trial, a diagnostic algorithm or largely approved treatment strategy is presently lacking in PACNS, the choice of a more or less aggressive immunosuppressive treatment (i.e. prolonged or short course of high-dose steroids with or without cyclophosphamide) should be tailored on the single case [13]. In addition, even if angiography by itself is not sufficient to make the specific diagnosis of vasculitis, biopsy results of angiographically abnormal vessels have been reported as normal [1], perhaps due to sampling error, patchy distribution of isolated CNS vasculitis, and a survey of
European neurologists diagnosing CNS vasculitis showed that only a minority sought biopsy proof of the diagnosis [14].

In conclusion, RCVS is a relative uncommon cause of intracerebral haemorrhage [2, 7]. Its definitive diagnosis is based on the reversible nature of the angiographic vasculiticlike lesions. Therefore, repeated cerebral angiograms together with clinical data are mandatory for the diagnosis, since leptomeningeal biopsy is negative in this setting, but false negative results may be also observed in PACNS [15, 16], and magnetic resonance imaging study may be not the exam of choice in acute and critical conditions. Delayed appearance of vasculitic-like lesions on angiography could suggest RCVS rather than PACNS. Early immunosuppressive treatment and type of regimen (less or more aggressive) should be based on the level of suspicion of RCVS or PACNS, and then modulated on the basis of the clinical outcome and the imaging study results in the short term.

\section{ACKNOWLEDGEMENT}

Declared none. 


\section{CONFLICT OF INTEREST}

Declared none.

\section{REFERENCES}

[1] Calabrese LH, Duna GF, Lie JT. Vasculitis in the central nervous system. Arthritis Rheum 1997; 40: 1189-201

[2] Calabrese LH, Dodick DW, Schwedt TJ, Singhal AB. Narrative Review: reversible cerebral vasoconstriction syndromes. Ann Int Med 2007; 146: 1463-44.

[3] Siva A. Vasculitis of the nervous system. J Neurol 2001; 248: 45168

[4] Haji-Ali RA, Furlan A, Abou-Chebel A, Calabrese LH. Benign angiopathy of the central nervous system: cohort of 16 patients with clinical course and long term follow up. Arthritis Rheum 2002: 47; 662-9

[5] Moskowitz SI, Calabrese LH, Weil RJ. Benign angiopathy of the central nervous system presenting with intracerebral haemorrhage. Surg Neurol 2007; 67: 522-7

[6] Salvarani C, Brown RD Jr, Calamia KT, et al. Primary central nervous system vasculitis presenting with intracranial hemorrhage. Arthritis Rheum 2011; 63: 3598-606

[7] Ansari SA, Rath TJ, Gandhi D. Reversible cerebral vasoconstriction syndromes presenting with subarachnoid hemorrhage: a case series. J Neurointerv Surg 2011; 3: 272-8

[8] Rinkel GJ, Wijdicks EF, Vermeulen M, Hageman LM, Tans JT, van Gijn J. Outcome in perimesencephalic (nonaneurysmal) subarachnoid hemorrhage: a follow-up study in 37 patients. Neurology 1990; 40: 1130-2

[9] Whiting J, Reavey-Cantwell J, Velat G, et al. Clinical course of nontraumatic, nonaneurysmal subarachnoid hemorrhage: a singleinstitution experience. Neurosurg Focus 2009; 26: E21

[10] Ducros A, Boukobza M, Porcher R, Sarov M, Valade D, Bousser MG. The clinical and radiological spectrum of reversible cerebral vasoconstriction syndrome. A prospective series of 67 patients. Brain 2007; 130: 3091-101

[11] Ghia D, Cuganesan R, Cappelen-Smith C. Delayed angiographic changes in postpartum cerebral angiopathy. J Clin Neurosci 2011; 18: 435-6

[12] Ducros A, Bousser MG. Reversible cerebral vasoconstriction syndrome. Pract Neurol 2009; 9: 256-67

[13] Salvarani C, Brown RD Jr, Hunder GG. Adult primary central nervous system vasculitis: an update. Curr Opin Rheumatol 2012; 24: 46-52.

[14] Scolding NJ, Wilson H, Hohlfeld R, Polman C, Leite I, Gilhus N. The EFNS cerebral vasculitis task force. The recognition, diagnosis and management of cerebral vasculitis: a European survey. Eur J Neurol 2002; 9: 343-7

[15] Spitzer C, Mull M, Rohde V, Kosinski CM. Non-traumatic cortical subarachnoid haemorrhage: diagnostic work-up and aetiological background. Neuroradiology 2005; 47: 525-31

[16] Zuccoli G, Pipitone N, Haldipur A, Brown RD Jr, Hunder G, Salvarani C. Imaging findings in primary central nervous system vasculitis. Clin Exp Rheumatol 2011; 29(1 Suppl 64): S104-S9.

(C) Quartuccio et al.; Licensee Bentham Open.

This is an open access article licensed under the terms of the Creative Commons Attribution Non-Commercial License (http://creativecommons.org/licenses/by-nc/ 3.0/) which permits unrestricted, non-commercial use, distribution and reproduction in any medium, provided the work is properly cited. 